\title{
« Un X peut en cacher un autre » : étude ethnosyntaxique
}

\author{
Bert Peeters \\ Macquarie University, Sydney \\ bert.peeters@mq.edu.au
}

Il fut une époque où, dans les gares de France, on pouvait passer d'un quai à l'autre en traversant les voies. Des panneaux qui disaient Un train peut en cacher un autre étaient installés là où la traversée des voies était permise. Installés par la SNCF, ces panneaux ont disparu avec l'avènement des passages souterrains ; cependant, tous ceux qui ont visité la France savent qu'à ce jour on continue à les trouver assez souvent (quoique moins souvent qu'autrefois) au bord de la route publique, près des passages à niveau, érigés de part et d'autre des voies ferrées. Les plus anciens de ces panneaux sont aujourd'hui assez rares pour être devenus des objets prisés. Lors d'une récente visite au célèbre Marché aux puces de Paris, près de la Porte de Clignancourt, l'auteur de ces lignes s'en est vu offrir un qu'il a failli acheter, ne fût-ce que le prix (€95) lui paraissait un peu excessif. Il aurait voulu emporter avec lui en Australie, pour le montrer à ses étudiants, ce panneau que, sauf erreur, on ne trouve nulle part ailleurs. Quelle meilleure façon, en effet, de se lancer dans une étude de la structure « Un X peut en cacher un autre » ? $^{1}$ Très répandue et instantanément reconnue, immédiatement et immanquablement associée à l'avertissement créé par la SNCF, cette structure constitue un objet d'étude privilégié de ce que, notamment à la suite de Wierzbicka (1979), nous avons appelé ailleurs l'ethnosyntaxe (Peeters 2009, 2010). Conformément à la définition que nous en avons donnée, le but de l'ethnosyntaxe est d'étudier, en invoquant des faits linguistiques aussi bien que non linguistiques, des structures syntaxiques productives propres à un univers culturel spécifique, en vue de découvrir si derrière ces structures se cachent des valeurs culturelles propres à cet univers. Il peut s'agir ou bien de valeurs déjà connues, que la démarche ethnosyntaxique permettra de mieux comprendre, ou bien de valeurs précédemment insoupçonnées - dont il s'agira ensuite de corroborer la réalité par d'autres moyens.

Dans un premier temps, nous évoquerons brièvement le sens original de l'avertissement de la SNCF et nous recourrons à la métalangue sémantique naturelle d'A. Wierzbicka et de C. Goddard pour en proposer une explicitation culturellement aussi neutre que possible $(§ 1)$. Deux développements qu'expliquent l'âge de la tournure Un train peut en cacher un autre et sa résonance familière retiendront ensuite notre attention. D'une part, au cours des années, un second sens s'est manifesté, qui coexiste aujourd'hui avec le sens original, celui qu'entendent véhiculer les panneaux de la SNCF. Ce développement fera l'objet de quelques remarques en fin d'exposé. D'autre part, sur le modèle du " train qui peut en cacher un autre », on a créé - et on continue à créer - toutes sortes d'énoncés dans lesquels, au nom train, se substitue un autre nom (voire un nom propre), ce qui permet, selon les circonstances, d'annoncer le sujet dont on va parler en réalité ( « je vais parler moins de $X$ que de $X$ ', que $X$ dérobe à la vue »), d'assurer la cohérence du discours («je viens de parler de X, maintenant je passe à X', que X dérobait à la vue »), voire de résumer ce qui vient d'être dit («j'ai parlé de X, mais plus encore de X', que X dérobe à la vue »). ${ }^{2}$ Le propre de ces expressions, on l'a dit, c'est qu'elles continuent à évoquer, d'une façon immédiate et incontestable, l'avertissement original. Autrement dit, il s'est développé une structure syntaxique productive. Cette productivité ne s'arrête pas à la possibilité d'avoir un nom plutôt qu'un autre dans la position sujet de la structure «Un X peut en cacher un autre ». L'un de nos objectifs est d'explorer l'étendue et les conséquences de la productivité d'une structure qui, au départ, n'avait rien de productif.

Mais d'où vient cette productivité ? C'est ce que contribueront à établir un nombre de témoignages explicites de la part d'observateurs internes et externes (\$2). Il y aura ensuite (§3) quelques indications sur la saillance culturelle de la structure qui sous-tend l'avertissement de la SNCF ; et ce n'est qu'après ce double exposé, à travers une analyse linguistique détaillée, qu'étendue et conséquences de la productivité 
de la formule seront envisagées (\$4). L'analyse fera ressortir un certain nombre de propriétés de la structure syntaxique productive qui se retrouvent en partie dans le dicton Un train peut en cacher un autre qui s'est développé à partir du sens original (§5). Les nombreux exemples qui seront cités permettront de préciser (§6) le sens de la structure, en métalangue sémantique naturelle, au-delà de l'impression, certes correcte, mais trop superficielle, qu'on a affaire à un avertissement; ils permettront également de formuler une hypothèse (\$7) relative aux valeurs culturelles propres à la langue-culture française. Rappelons que la formulation d'hypothèses de ce genre, à partir de l'étude de structures syntaxiques productives, est le but ultime de la démarche ethnosyntaxique telle que nous l'avons définie ci-dessus. Une étude ethnoaxiologique, qu'il nous est impossible d'entreprendre ici, devra ensuite prouver à partir d'autres faits, linguistiques aussi bien que non linguistiques, le bien-fondé de l'hypothèse.

\section{1 « Un train peut en cacher un autre »}

Précisons d'abord, en quelques mots, le message global véhiculé par l'avertissement qu' « un train peut en cacher un autre ». Il s'agit de rappeler aux automobilistes, aux cyclistes, aux piétons, bref à tous ceux qui ont besoin de traverser la voie ferrée que, lorsqu'un train vient de passer et qu'ils se préparent à poursuivre leur voyage, mieux vaut vérifier si, entretemps, un autre train n'arrive pas de la même direction ou de la direction opposée. Le but du message est d'inciter les usagers de la voie publique à la prudence : «Ne traversez pas sans vous assurer que la voie est libre...».

Les choses se compliquent quand on passe du message à ses éléments constitutifs. Certes, nous savons tous, pour l'avoir appris, ce qu'est un train - et nous savons tous ce que veut dire qu'une chose en cache une autre. Mais quel est au juste le sens du verbe pouvoir, non pas en général mais dans le contexte d'un train qui peut en cacher un autre ?3 Il y a des lectures qui peuvent être aussitôt exclues; ce sont notamment celles qui relèvent de la modalité illocutoire telle que la définit Vetters (2007), et que cet auteur illustre à l'aide des exemples suivants tirés de la littérature savante sur le sujet :

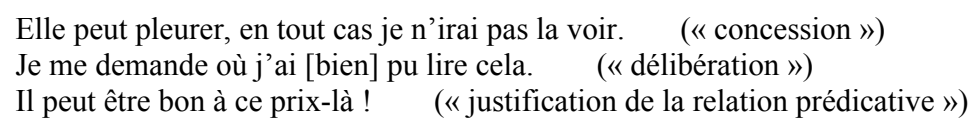

Il est clair que la tournure Un train peut en cacher un autre n'implique ni concession ni délibération de la part du locuteur, et que celui-ci ne cherche pas non plus à justifier la relation prédicative entre le sujet $u n$ train et le prédicat en cacher un autre. Peuvent également être exclues les lectures «permission » et «possibilité matérielle ». ${ }^{4}$ Trois autres lectures demeurent a priori possibles. La première relève, avec les lectures «permission» et "possibilité matérielle», de la modalité radicale : c'est la "capacité » (les dimensions du train). Les deux autres relèvent de la modalité épistémique : ce sont l' «éventualité » (un train en cache peut-être un autre) et la « sporadicité » (un train en cache parfois un autre).

Comment se décider? Avant de répondre à cette question, posons-en une autre, à savoir : faut-il se décider ? Qu'importe la lecture, pourvu que le message passe... Il est vrai qu'il ne passe pas toujours les statistiques accidentelles le confirment - mais il passe certainement bien plus souvent que non. À cela s'ajoute qu'au moment de créer la tournure Un train peut en cacher un autre les responsables de la SNCF ne se sont surement pas posé la question de savoir s'ils cherchaient à mettre le public en garde contre les dimensions du train, ou les circonstances, ou la présence éventuelle ou sporadique d'un autre train. Ils ont tout simplement... voulu mettre le public en garde. Pour ce faire, ils se sont servis d'un verbe qui se prêtait à toutes ces lectures, et à d'autres : un verbe suffisamment vague en quelque sorte. Aussi n'est-il pas nécessaire de se perdre dans toutes les fines distinctions faites par des générations de linguistes, d'autant plus que ces distinctions reposent sur une terminologie sémantiquement complexe qui revient à essayer d'expliquer ce qui est sémantiquement simple en termes de ce qui est sémantiquement complexe. Le verbe pouvoir ne se laisse pas définir de façon plus simple; en revanche, les distinctions qu'on a coutume d'effectuer en son sein peuvent - et doivent - être définies en termes du primitif sémantique POUVOIR qui, lui, fait partie de la métalangue sémantique naturelle développée aux cours des quarante 
dernières années, d'abord par A. Wierzbicka, ensuite par toute une équipe de linguistes travaillant en collaboration étroite avec A. Wierzbicka et avec celui qui est devenu à partir de la fin des années quatrevingts son bras droit, C. Goddard (voir par exemple, parmi les textes les plus récents, Wierzbicka 2006, Peeters 2006, Goddard 2008, Peeters 2010). La métalangue sémantique naturelle (MSN), dont il existe autant de versions rigoureusement isomorphes qu'il y a de langues naturelles, est une langue qui, comme toutes les langues naturelles, a un lexique et une grammaire (contrairement à un grand nombre d'autres métalangues). Seulement, on l'aura deviné, ce lexique et cette grammaire sont fortement particularisés, sans quoi l'isomorphie des différentes versions ne saurait être garantie. Tout d'abord, les primitifs sémantiques qui constituent le lexique de la MSN ne sont pas des lexèmes au sens propre de ce terme : il s'agit plutôt de sens lexicalisés (sous forme de mots, de morphèmes ou de phrasèmes) dans l'ensemble des langues naturelles. Les primitifs sont donc des universaux et POUVOIR, en tant que primitif sémantique, est un élément universel. Les recherches empiriques entreprises au sein de ce qu'on appelle aujourd'hui l'approche MSN permettent de dire avec un fort degré de probabilité qu'il existe, dans toutes les langues naturelles, un mot (ou un morphème ou un phrasème) qui correspond au primitif représenté dans la version anglaise de la MSN sous forme de CAN et dans la version française sous forme de POUVOIR. Les lexicalisations anglaises et françaises des primitifs sémantiques figurent dans les tableaux ci-dessous.

Tableau 1 - Le lexique de la MSN, classé en rubriques (version anglaise)

\begin{tabular}{|l|l|}
\hline I, YOU, SOMEONE, SOMETHING, PEOPLE, BODY & Substantifs \\
\hline KIND, PART & Substantifs relationnels \\
\hline THIS, SAME, ${ }^{5}$ OTHER & Déterminants \\
\hline ONE, TWO, MUCH / MANY, SOME, ALL & Quantificateurs \\
\hline GOOD, BAD & Évaluateurs \\
\hline BIG, SMALL & Descripteurs \\
\hline THINK, KNOW, WANT, FEEL, SEE, HEAR & Prédicats mentaux \\
\hline SAY, WORDS, TRUE & Discours \\
\hline DO, HAPPEN, MOVE, TOUCH & $\begin{array}{l}\text { Actions, évènements, } \\
\text { mouvement, contact }\end{array}$ \\
\hline BE (SOMEWHERE), THERE IS, HAVE, BE (SOMEONE / SOMETHING) & $\begin{array}{l}\text { Emplacement, existence, } \\
\text { possession, spécification }\end{array}$ \\
\hline LIVE, DIE & Vie et mort \\
\hline $\begin{array}{l}\text { WHEN, NOW, BEFORE, AFTER, A LONG TIME, } \\
\text { A SHORT TIME, FOR SOME TIME, MOMENT }\end{array}$ & Temps \\
\hline WHERE, HERE, ABOVE, BELOW, FAR, NEAR, SIDE, INSIDE & Espace \\
\hline NOT, MAYBE, CAN, BECAUSE, IF & Concepts logiques \\
\hline VERY, MORE & $\begin{array}{l}\text { Intensificateur et } \\
\text { augmentateur }\end{array}$ \\
\hline LIKE & Similarité \\
\hline
\end{tabular}


Tableau 2 - Le lexique de la MSN, classé en rubriques (version française)

\begin{tabular}{|c|c|}
\hline JE, VOUS, ${ }^{6}$ QUELQU'UN, QUELQUE CHOSE, GENS, CORPS & Substantifs \\
\hline TYPE, PARTIE & Substantifs relationnels \\
\hline CE, MÊME, AUTRE & Déterminants \\
\hline UN, DEUX, BEAUCOUP, CERTAINS, ${ }^{7}$ TOUT & Quantificateurs \\
\hline BIEN, MAL & Évaluateurs \\
\hline GRAND, PETIT & Descripteurs \\
\hline PENSER, SAVOIR, VOULOIR, SENTIR, VOIR, ENTENDRE & Prédicats mentaux \\
\hline DIRE, MOTS, VRAI & Discours \\
\hline FAIRE, ARRIVER, BOUGER, TOUCHER & $\begin{array}{l}\text { Actions, évènements, } \\
\text { mouvement, contact }\end{array}$ \\
\hline $\begin{array}{l}\text { ÊTRE (QUELQUE PART), IL Y A, } \\
\text { AVOIR, ÊTRE (QUELQU'UN / QUELQUE CHOSE) }\end{array}$ & $\begin{array}{l}\text { Emplacement, existence, } \\
\text { possession, spécification }\end{array}$ \\
\hline VIVRE, MOURIR & Vie et mort \\
\hline $\begin{array}{l}\text { QUAND, MAINTENANT, AVANT, APRÈS, LONGTEMPS, } \\
\text { PEU DE TEMPS, POUR QUELQUE TEMPS },{ }^{8} \text { MOMENT }\end{array}$ & Temps \\
\hline OÙ, ICI, AU-DESSUS, AU-DESSOUS, LOIN, PRÈS, CÔTÉ, DANS & Espace \\
\hline NE ... PAS, PEUT-ÊTRE, POUVOIR, À CAUSE DE, SI & Concepts logiques \\
\hline TRÈS, PLUS & $\begin{array}{l}\text { Intensificateur et } \\
\text { augmentateur }\end{array}$ \\
\hline COMME & Similarité \\
\hline
\end{tabular}

D'aucuns s'étonneront que la liste des primitifs soit tellement limitée. À vrai dire, au départ, elle l'était bien plus encore : dans Wierzbicka (1972), ouvrage traduit du polonais, l'inventaire ne comportait qu'une douzaine d'éléments. C'est qu'il y avait - et il y a toujours - un autre critère d'inclusion (ou d'exclusion), qui s'ajoute à celui de l'universalité, à savoir celui de la simplicité sémantique. Pour qu'un élément lexical soit inclus dans le lexique de la MSN, il faut que, dans l'emploi qu'on en fera dans la métalangue, il soit sémantiquement simple, c'est-à-dire indivisible et indéfinissable - à moins d'avoir recours à des termes plus techniques (ce qui est contraire à l'idée que l'acte de définir revient à rendre simple ce qui ne l'est pas). Les primitifs sémantiques ont été appelés ainsi précisément parce qu'ils sont indéfinissables et indivisibles. Les définitions proposées au départ pour un certain nombre d'éléments qui, à l'heure actuelle, font partie de la liste se sont révélées inappropriées et ont été abandonnées. C'est ainsi qu'on en est arrivé, chemin faisant, à l'inventaire actuel.

Il est important de préciser que, souvent, les lexicalisations des primitifs se prêtent à des usages qui ne sont pas universels, et dès lors bannis de la métalangue, et que ces usages peuvent différer d'une langue à l'autre. Les sens qu'on admet sont illustrés à l'aide d'une ou de plusieurs phrases ou contextes dits canoniques, qui exemplifient l'usage qu'on peut faire des éléments lexicaux constitutifs du lexique de la métalangue. Voici quelques exemples de phrases canoniques; elles consistent pour la plupart, mais pas exclusivement, de primitifs sémantiques : 


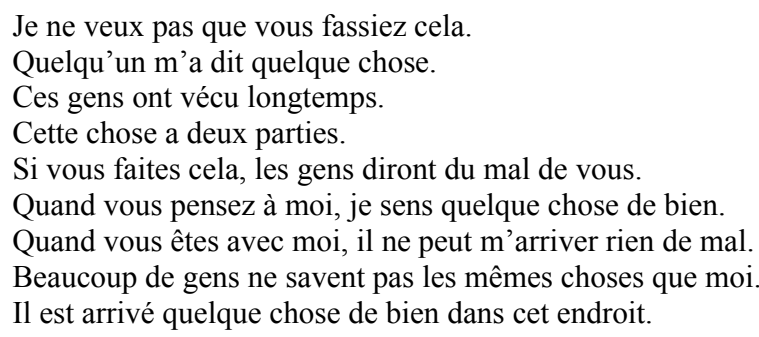

Les phrases canoniques permettent en outre de préciser les propriétés grammaticales et syntaxiques de chacun des primitifs. L'hypothèse, sous-tendue par des recherches empiriques extensives, est que chacun des primitifs a un nombre limité de propriétés syntaxiques tout aussi universelles que les primitifs euxmêmes. Autrement dit, si le lexique de la métalangue sémantique naturelle est universel, sa grammaire l'est tout autant. Sans entrer dans le détail, précisons simplement que les propriétés syntaxiques des primitifs sont de plusieurs types. Certaines d'entre elles précisent quels sont les primitifs que l'on peut simplement juxtaposer: le déterminant CE peut ainsi être suivi du substantif GENS (CES GENS), et de la même façon on peut avoir des combinaisons tels que QUELQU'UN D'AUTRE, BEAUCOUP D'ENDROITS, LOIN D'ICI (où D' est une simple ligature), mais aussi DEUX PARTIES, LA MÊME CHOSE, etc. D'autres propriétés concernent les soi-disant valences syntaxiques de certains des primitifs: contrairement à TOUT, BEAUCOUP admet une option partitive (BEAUCOUP DE CES GENS, mais non pas *TOUS DE CES GENS); dans le cas du verbe FAIRE, on peut avoir QUELQU'UN FAIT QUELQUE CHOSE, QUELQU'UN FAIT QUELQUE CHOSE À QUELQUE CHOSE, QUELQU'UN FAIT QUELQUE CHOSE À QUELQU'UN, QUELQU'UN FAIT QUELQUE CHOSE À QUELQU'UN / QUELQUE CHOSE AVEC QUELQUE CHOSE, QUELQU'UN FAIT QUELQUE CHOSE AVEC QUELQU'UN. Finalement, il y a des propriétés qui dépassent le niveau propositionnel: le primitif SAVOIR peut ainsi être suivi d'une subordonnée (SAVOIR QUE P). ${ }^{9}$

De par le caractère universel des primitifs et de leurs propriétés combinatoires, le lexique et la grammaire de chacune des versions de la métalangue sémantique naturelle permettent à la langue dont ils relèvent d'assumer le rôle de métalangue universelle et dès lors culturellement neutre, d'outil descriptif rigoureux et d'une clarté maximale déployé pour expliciter tout ce qui est culturellement spécifique, qu'il s'agisse de mots, d'expressions, de normes communicatives, de structures syntaxiques, de valeurs culturelles, et ainsi de suite. Le «sens SNCF » de la tournure Un train peut en cacher un autre peut être explicité comme suit :

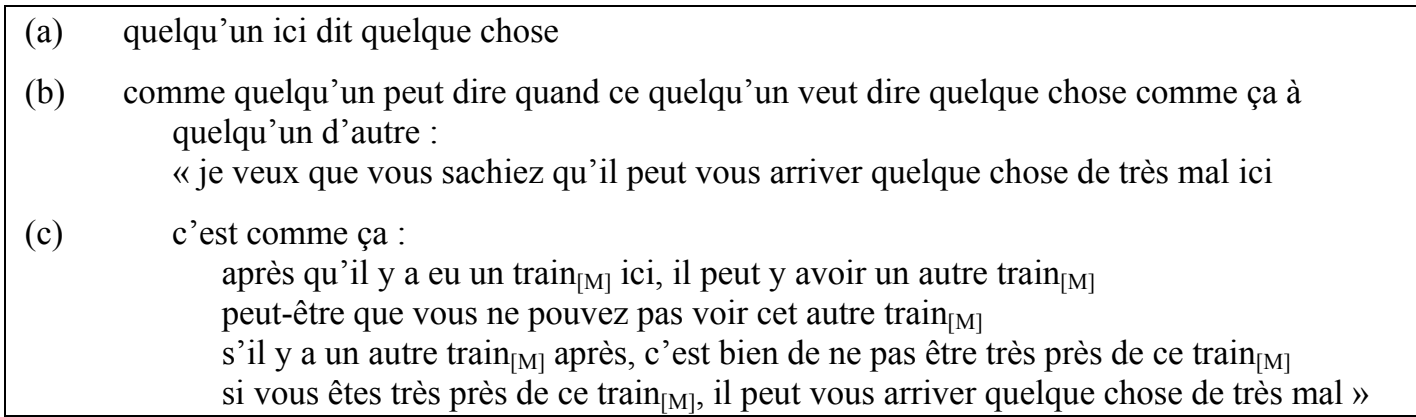

Le volet (a) pose d'emblée que la tournure Un train peut en cacher un autre est un acte de parole lié à un endroit spécifique. Le volet (b) précise qu'il s'agit d'un acte de parole particulier, que l'on a coutume de désigner comme un avertissement (ou une mise en garde). Avertir quelqu'un, c'est dire à la personne à qui on s'adresse qu'elle doit être au courant d'un risque qu'elle court. Pour ce faire, on peut s'exprimer de plusieurs façons, dont celle qui figure dans l'explicitation. Le volet (c) fournit des détails sur la nature du risque couru. Afin d'en garantir la lisibilité, nous maintenons le mot train et y conférons le statut de molécule sémantique (d'où la notation $\operatorname{train}_{[M]}$ ). On a besoin de cette molécule relativement productive non seulement pour décrire le sens de l'avertissement de la SNCF, mais aussi pour décrire celui de mots 
tels que locomotive, wagon, omnibus, gare, passage à niveau, chemin de fer et celui des nombreuses expressions figurées où se retrouve le mot train. Signalons, à titre d'exemple : à fond de train, aller grand train (allusions à la vitesse); faire un train d'enfer (allusion au bruit); prendre le train en marche (allusion à la tardivité); être passé sous un train (allusion à la défiguration); comme une vache qui regarde passer un train (allusion à la passivité, la stupidité). ${ }^{10}$

\section{$2 \quad$ Productivité de la structure : témoignages explicites}

Comment expliquer qu'un texte figurant sur des panneaux de signalisation ait pu donner lieu à une structure syntaxique productive de la langue contemporaine, pour devenir « une de ces phrases définitives qui ont le plus inspiré de titres d'articles » (François Kermoal, Stratégies, 24 janvier 2008) ? ${ }^{11}$ Ce n'est pas une simple question de fréquence: la présence, jusque dans un passé relativement récent, des panneaux de la SNCF près de tous les passages à niveau a certainement contribué à ce développement, mais ne peut pas, à lui seul, en être tenu responsable. Il y a d'innombrables autres textes figurant sur d'innombrables autres panneaux (ou ailleurs) qui n'ont pas connu le même sort. La différence entre ceuxci et celui-là réside dans l'impact qu'ont eu les panneaux de la SNCF. On a beaucoup parlé de trains qui peuvent en cacher d'autres. L'avertissement n'est pas resté lettre morte. Il surgit régulièrement dans la presse écrite quand, malgré tout, un train a fini par happer et tuer des victimes innocentes. En outre, il occupe une place de premier plan dans les campagnes de sensibilisation incitant les usagers de la route à la prudence lors d'une traversée de passages à niveau. Autrement dit, il est devenu célèbre. On l'a souvent souligné, notamment à l'aide de ce mot-là, parfois dans des contextes où il n'est nullement question de trains :

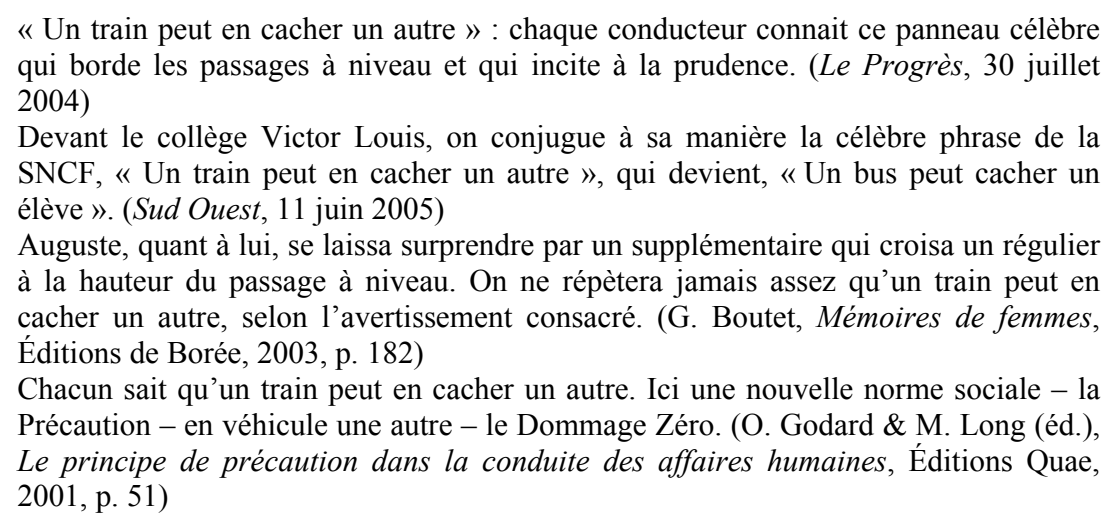

Un grand nombre d'observateurs externes se sont exprimés de façon analogue, dans des textes portant sur les sujets les plus différents. On notera, dans les extraits qui suivent, les adjectifs anglais familiar et famous, les tournures espagnoles muy usual 'très habituel' et conoces bien 'on connait bien', et le tour néerlandais dat weet iedereen in Frankrijk 'cela, tout le monde en France le sait' :

«Un train peut en cacher un autre » - one train can hide another - reads the familiar warning sign at railroad crossings in France. So, too, can one TGV. (J. Meunier, On the fast track, Praeger, 2002, p. 9)

One train can hide another, goes the famous warning sign at French railway junctions. The same might be said for Indian entrepreneurs. (Financial Times, 26 janvier 2007)

La situación de los hombres maltratados por sus parejas femeninas me hace pensar en una advertencia muy usual en los cruces entre la carretera y los rieles, en Francia : «!Cuidado $;$ !Un tren puede ocultar a otro; ». El grave problema de violencia contra las mujeres cubre con una sombra de oprobio y silencio el caso inverso. (El comercio, 16 mars 2008)

Conoces bien la leyenda francesa : Un train peut en cacher un autre. El tránsito de las noticias : unas tapan otras. (El Mundo, 7 mars 2009) 
Un train peut en cacher un autre. Dat weet iedereen in Frankrijk. (De Morgen, 23 novembre 2007)

Les renvois explicites à la formule de la SNCF dans des contextes autres que la traversée des voies ferrées sont devenus monnaie courante, sans qu'il soit nécessaire d'attirer l'attention sur sa renommée.

\begin{abstract}
Le panneau de signalisation « Un train peut en cacher un autre » typiquement français est parfaitement adapté à la situation du chemin de fer en Europe... Derrière la réalité concrète d'un train qui circule sur les rails du Vieux Continent, se cachent des accords ferroviaires particulièrement complexes. (Le Monde, 22 septembre 1998)

En paraphrasant la mention en forme d'avertissement que l'on peut lire encore à certains passages à niveau : "Attention, un train peut en cacher un autre », on pourrait écrire : "Attention, un horizon peut en cacher un autre ", et c'est souvent ce qui se produit ; le paysage acquiert ainsi une profondeur infinie. (J. Orizet, Les aventures $d u$ regard, Jean-Pierre Huguet, 1999, p. 145) ${ }^{12}$
\end{abstract}

Plus souvent que non, quand l'avertissement de la SNCF est explicitement mise en rapport avec une autre formule du type «Un X peut en cacher un autre», on voit apparaitre des connecteurs tels que comme, de même que, de la même manière que, à la manière de, si $:^{13}$

Petite réserve : un objectif ultime en cache toujours un autre plus lointain, comme un train peut en cacher un autre. (J. Spinetta, La pratique de l'illumination et la science de l'immortalité par les méditations, Fernand Lanore, 2006, p. 42)

De même qu'un train peut en cacher un autre, une disparition peut en cacher une autre. (Le Monde, 13 juillet 2003)

De la même manière qu'un train peut en cacher un autre, il faut croire qu'un artiste peut aussi en cacher un autre. (Le Progrès de Lyon, 23 février 2005)

À la manière des panneaux ferroviaires qui prévenaient naguère qu' " un train peut en cacher un autre », la guerre à la drogue menée par les États-Unis cache bien la volonté de poursuivre une politique de présence en Amérique latine. (Philippe Marchesin, Les nouvelles menaces, Karthala, 2001, pp. 175-176)

C'est l'un des casse-tête des transports : si un train peut en cacher un autre, une modernisation peut (souvent) en entrainer une autre... (Sud Ouest, 19 septembre 2006)

Parfois, le lien entre les deux formules est établi d'une façon plus subtile :

Comme à la SNCF, une image peut en cacher une autre ou des paroles une image et vice versa. (Le Monde Diplomatique, mai 2001)

Un mot est parfois comme un train : il peut en cacher un autre. (Le Monde, 22 octobre 2006)

De façon plus générale, on peut dire que ceux qui recourent à la structure «Un X peut en cacher un autre» (ou qui l'évoquent tout simplement), tout en renvoyant à l'avertissement original, témoignent explicitement de sa productivité. Il est clair que le panneau de la SNCF a parlé à l'imagination des Français. Le texte qui y figure occupe une place de premier plan dans la psyché française. Ce n'est pas une surprise que Pierre Georges, en évoquant avec une certaine nostalgie le «monde d'autrefois », l'ait fait de la façon suivante :

Autrefois, on parle du $\mathrm{XX}^{\mathrm{e}}$ siècle, [la vie] s'ordonnait autour de quelques principes de précaution simples et facilement mémorisables: un train peut en cacher un autre, è pericoloso sporgersi, te penche pas au balcon, fais du bruit quand tu t'aventures dans les terrains à vipères, traverse dans les clous, mâche bien avant d'avaler. Etc., etc. (Le Monde, 29 mars 2002)

Il est significatif que le tout premier des «principes de précaution » mentionnés soit celui du train qui peut en cacher un autre. Il s'agit de toute évidence d'un texte bien connu et instantanément reconnu. 


\section{Saillance culturelle}

La structure « Un X peut en cacher un autre » jouit d'une saillance culturelle considérable, dans la mesure où elle occupe une place importante dans la presse, à la télévision, dans les ouvrages parus en librairie, dans les slogans, et enfin sur les affiches et les tracts.

\subsection{Dans la presse}

Parmi les «phrases définitives qui ont le plus inspiré de titres d'articles» (cf. §2), il y en a, selon F. Kermoal, deux qui viennent immédiatement à l'esprit : ce sont l'ancienne devise de Paris-Match, à savoir Le poids des mots, le choc des photos, et l'avertissement de la SNCF, Un train peut en cacher un autre. ${ }^{14}$ Les titres de presse ne constituent qu'un seul domaine parmi plusieurs autres où ce dernier a laissé une marque désormais indélébile. Les occurrences de la structure «Un X peut en cacher un autre » ne sont jamais très loin. Même en l'espace d'un an, on en trouve littéralement des dizaines. Ce n'est pas le moment d'entrer dans le détail ; des listes assez complètes figurent dans le volet consacré à la productivité de la structure « Un X peut en cacher un autre » $(\S 4)$.

\section{2 À la télévision}

L'idée ne manquait pas d'originalité, mais les téléspectateurs ne l'ont pas aimée - pas assez pour que France 2 continue la diffusion de l'émission quotidienne Une surprise peut en cacher une autre au-delà du 19 décembre 2008. Lancé moins de trois mois auparavant, le lundi 29 septembre, le programme voulait d'abord créer des «chaines de surprises hebdomadaires ». Il s'agissait de donner à quelqu'un la chance de remercier quelqu'un d'autre en lui faisant une surprise (par exemple, une rencontre avec une idole, une fête, le réaménagement d'une maison). C'était l'émission du lundi soir. Le mardi, la deuxième personne devait en surprendre une troisième, et ainsi de suite jusqu'au jeudi. Les quatre "bénéficiaires » se retrouvaient alors le vendredi pour surprendre l'initiateur. Il y a eu au total quatre "boucles »; fin octobre, le programme a été interrompu pour être relancé le 10 novembre avec une formule révisée. Chaque émission serait dorénavant autonome : chacun des participants allait devoir surprendre quelqu'un d'autre après avoir bénéficié lui-même d'une surprise initiale. Le changement de formule n'a pas eu l'effet escompté ; cependant, ce qui est significatif de notre point de vue, c'est le choix du titre.

Au cours de ces dernières années, «Un X peut en cacher un autre » a également inspiré le titre de divers épisodes de séries policières et autres, de reportages, d'émissions pour enfants, etc.

\subsection{Dans les ouvrages parus en librairie}

Parmi les titres de librairie pertinents sortis depuis 2000, il y a un grand nombre de textes non fictionnels (ouvrages d'érudition, essais, ouvrages de vulgarisation, journalisme d'investigation); ils trouvent en général moins de lecteurs que les récits fictionnels, parmi lesquels on trouve des romans, des albums de poèmes illustrés, des livres pour enfants et des bandes dessinées. Le plus original des romans est peut-être celui d'I. Morot-Sir qui, dans Attention! Un train peut en cacher un autre... (Publibook, 2005), raconte l'histoire d'une femme qui, pendant qu'elle s'est retirée dans les toilettes d'un train, est projetée contre la paroi quand le wagon où elle se trouve, tout à coup, secoue violemment. Quand elle ouvre la porte, elle se trouve dans un train entièrement différent, d'une époque révolue : elle vient de faire un voyage dans le temps. À signaler aussi, les deux recueils de poèmes de F. David : ils s'intitulent Un éléphant peut en cacher un autre et Un loup peut en cacher un autre (Éditions Sarbacane, 2005, 2006). Retenons finalement, dans le genre "littérature pour enfants », un récit de M. Amaury intitulé Un cheval peut en cacher un autre (Flammarion, 2004), et une bande dessinée de Tarek, L. Chouin et C. Bouchard, Un derviche peut en cacher un autre (Emmanuel Proust, 2006). 


\subsection{Dans les slogans}

À l'occasion de la quatrième journée nationale de lutte contre l'herpès, organisée le 6 novembre 2004 sous le patronage du Haut comité de la santé publique, un slogan fut adopté : c'était, on s'y attend, Un herpès peut en cacher un autre. Le slogan - en même temps le titre d'un article paru dans le numéro 105 (2004) de la Revue de l'infirmière et celui de plusieurs articles non signés dans la presse quotidienne (notamment dans Le Bien Public du $1^{\text {er }}$ novembre 2004) - cherchait à attirer l'attention du public sur le fait que 20 à $30 \%$ des herpès génitaux ont pour origine un simple bouton de fièvre, et que les infections herpétiques n'affectent pas seulement le visage et les parties génitales: elles peuvent intervenir sur plusieurs autres parties du corps, dont le tronc, les bras, les doigts, les jambes et l'œsophage.

\subsection{Sur les affiches et les tracts}

Parmi les affiches qui exploitent la structure « Un X peut en cacher un autre », il faut en particulier retenir l'un des lauréats du concours « Drogues : plaisirs, risques, dépendances » organisé en 2003 par le CRIPS (Centre régional d'information et de prévention du sida) de l'île-de-France, à l'occasion d'un festival international de l'affiche et des arts graphiques. L'affiche en question, réalisée par un étudiant en graphisme, avait été créée afin d'appuyer une campagne dont le but était d'engager la lutte contre l'addiction au cannabis. Elle montre, en noir sur un fond jaune vif, une main qui offre une cigarette et un bras qui se tend pour la prendre. Le texte imprimé sur l'affiche est Attention, un train peut en cacher un autre. Selon Lydie Desplanques, du CRIPS Île-de-France (courriel à l'auteur daté du 4 mars 2009), il n'y a pas d'interprétation définie : « la formule est volontairement assez imprécise pour que les personnes qui la lisent s'interrogent dessus, en parlent autour d'eux pour essayer de la comprendre, confrontent leurs représentations... ». Il existerait néanmoins deux interprétations assez probables, plus ou moins en rapport avec le sens originel de la formule : la phrase « fait réfléchir sur la perte d'attention que peut entrainer la consommation de cannabis » (les deux trains représentent dans ce cas-là n'importe quel danger, en particulier les dangers liés aux transports); ou bien «le premier train symbolise une consommation de cannabis plutôt exceptionnelle et l'autre train représente des consommations de cannabis imprévues, plus nombreuses, plus fréquentes, voire des consommations d'autres produits ».

Par ailleurs, un tract du Parti Communiste Français (PCF), diffusé en mai 2008, s'intitule Constitution: Attention, cette réforme peut en cacher bien d'autres! Le PCF voulait, en la diffusant, protester contre les réformes constitutionnelles du président de la République, Nicolas Sarkozy.

\section{$4 \quad$ Étude linguistique approfondie}

Un grand nombre d'exemples de la structure «Un X peut en cacher un autre » ont été cités dans ce qui précède - et plusieurs dizaines d'autres vont suivre. Aucun commentaire linguistique n'a cependant été fourni jusqu'ici, hormis un renvoi aux connecteurs utilisés quand une tournure qui s'inspire de l'avertissement original se trouve utilisée en même temps que ce dernier. Ce quatrième volet est consacré à une étude linguistique approfondie, qui examinera l'étendue de la productivité de la structure à l'étude de même que les conséquences qui en résultent.

\section{1 Étendue de la productivité}

C'est au niveau du temps verbal et à celui du sujet et du complément d'objet direct que se mesure la véritable productivité de la structure "Un X peut en cacher un autre ». Dans la grande majorité des cas, l'élément qui occupe la place du X est un simple nom. Il n'y a presque jamais d'adjectif; il n'y en a pas non plus dans l'avertissement de la SNCF. En théorie, la présence d'un adjectif permettrait de s'éloigner davantage de la version originale : plutôt que de dire Une bonne nouvelle peut en cacher une autre (Le Progrès de Lyon, 24 novembre 2007 ; cf. aussi le premier des trois exemples à l'imparfait ci-dessous), on 
pourrait avoir Une bonne nouvelle peut en cacher une mauvaise ou bien Une bonne nouvelle peut en cacher une moins bonne, etc. Mais cela n'arrive pratiquement jamais : le texte des panneaux de la SNCF maintient pour ainsi dire son emprise. Il suffit pour s'en convaincre de faire un petit test autour de soi. Glissez dans vos conversations une occurrence de la structure «Un X peut en cacher un autre », avec n'importe quel nom à la place du X, et arrêtez-vous après l'infinitif : votre interlocuteur suppléera quasi immanquablement les mots un autre ou une autre, selon le cas.

Le simple nom qui prend la place du X peut continuer à évoquer la SNCF, ou bien le panneau ou le texte eux-mêmes, et ce de diverses façons : ${ }^{15}$

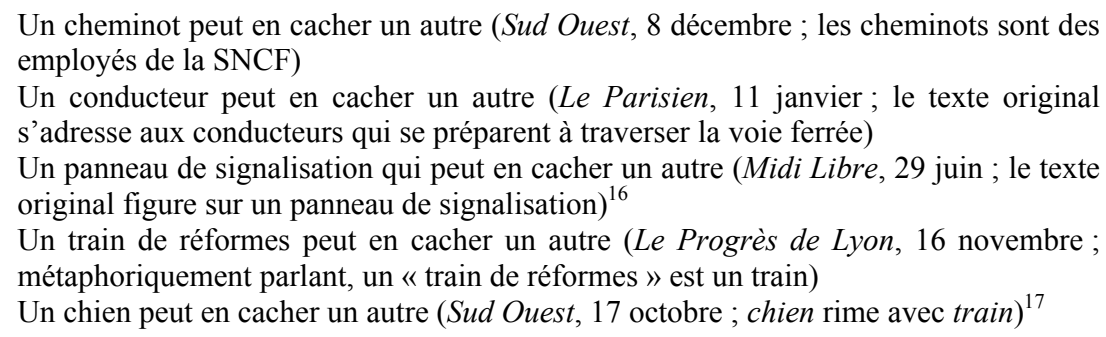

Il est fréquent aussi que le nom en question désigne un autre moyen de transport ou bien quelque chose qui, tel un train, se fait remarquer à cause de ses dimensions physiques (le deuxième exemple ci-dessous relève des deux catégories à la fois) :

Un taxi peut en cacher un autre (Sud Ouest, 27 septembre)

Un avion peut en cacher un autre (Le Nouvel Économiste, 19 juillet)

Un bâtiment peut en cacher un autre (Le Figaro, 17 janvier)

Quant aux créations que voici, elles aussi restent au moins partiellement motivées :

Un rond-point peut en cacher un autre (Sud Ouest, 7 février ; les ronds-points, comme les trains, créent des situations à risques pour les automobilistes)

Un (vieux) pont peut en cacher un autre (Le Progrès de Lyon, 24 juillet, 31 aout ; même remarque pour les ponts, surtout s'ils sont vieux)

En revanche, dans la plupart des cas, il n'y a simplement aucun rapport. Le nom sujet peut être celui d'un objet (palpable ou non) :

Une image peut en cacher une autre (L'Express, 12 avril)

Assainir : Attention, un chiffre peut en cacher un autre (Midi Libre, 28 octobre)

Une gerbe peut en cacher une autre (Sud Ouest, 30 avril ; La Charente Libre, 1

novembre)

d'un endroit (noms communs aussi bien que noms propres) :

Un Bazouges peut en cacher un autre (Ouest France, 21 mars ; Bazouges La Perouse se trouve en Normandie)

Un chantier peut en cacher un autre (Le Progrès de Lyon, 14 avril)

Un arrêt peut en cacher un autre (Centre Presse, 11 septembre)

Une ville peut en cacher une autre (Le Parisien, 22 décembre)

d'une activité humaine (y compris les actes de parole) :

Une mini-entreprise peut en cacher une autre (Le Progrès de Lyon, 22 mars)

Un vol peut en cacher un autre (Sud Ouest, 24 mars)

Une campagne peut en cacher un autre (Centre Presse, 17 avril)

À Lyon, une élection peut en cacher une autre (Le Parisien, 7 juin)

Un divorce peut en cacher un autre (Sud Ouest, 25 juin)

Un éloge peut en cacher un autre (Le Monde, 30 novembre) 
d'une situation inquiétante :

Une décharge peut en cacher une autre (Sud Ouest, 30 mars)

Un problème peut en cacher un autre (Le Progrès de Lyon, 17 septembre)

Une catastrophe peut en cacher une autre (Ouest France, 6 octobre)

Banques : une « anomalie » peut en cacher une autre (Le Figaro, 23 novembre)

Une « caisse noire » peut en cacher une autre (L'Humanité, 12 décembre)

d'un animal :

Un coq peut en cacher un autre (Le Progrès de Lyon, 15 septembre)

d'un être humain identifié sur la base de son sexe, de son âge, de son rôle dans la société, d'une distinction :

Un homme peut en cacher un autre (Le Figaro, 15 janvier)

Une femme peut en cacher une autre (Centre Presse, 24 octobre)

Une centenaire peut en cacher une autre (Le Progrès de Lyon, 21 avril)

Un père peut en cacher un autre... (L'Humanité, 7 avril)

Un juge peut en cacher un autre (Le Vif / L'Express, 23 mars)

Un maire peut en cacher un autre... (Centre Presse, 19 septembre)

Un auteur peut en cacher un autre (L'Express, 15 mars)

Un consommateur peut en cacher un autre! (Les Échos, 27 décembre)

Un candidat peut en cacher un autre (Sud Ouest, 5 juillet)

Attention, un créancier peut en cacher un autre (Enjeux Les Échos, 1 juillet)

Une Miss peut en cacher une autre (Le Progrès de Lyon, 20 mars)

d'un être humain identifié à l'aide d'un nom propre (prénom et/ou nom de famille) :

Un Claude peut en cacher un autre (Libération, 29 mars)

Une Véronique peut en cacher une autre (Le Progrès de Lyon, 31 mai)

Un Laurent peut en cacher un autre (Le Progrès de Lyon, 16 juillet)

Un Cohn-Bendit peut en cacher un autre ( $L$ 'Indépendant du Midi, 8 février)

Un Delanoë peut en cacher un autre (Le Parisien, 5 septembre)

Une Kirchner peut en cacher une autre (Sud Ouest, 28 octobre)

Un Poutine peut en cacher un autre (Midi Libre, 29 novembre)

Un Alain Landais peut en cacher un autre (Le Parisien, 16 juin)

d'un ensemble d'humains :

Une DDE peut en cacher une autre (Sud Ouest, 9 janvier; DDE = Direction départementale de l'équipement)

Un sommet peut en cacher un autre (Le Journal des Finances, 8 septembre)

Dans certains cas, l'écart formel par rapport à l'avertissement de la SNCF est plus considérable que dans les exemples énumérés jusqu'ici. C'est ce qui arrive, par exemple, quand le sujet et l'objet se mettent tous deux au pluriel, comme dans les titres de presse que voici :

Des cheminées peuvent en cacher d'autres (Le Progrès de Lyon, 27 octobre 2004)

Des travaux qui peuvent en cacher d'autres (Le Progrès de Lyon, 11 mars 2006)

Il se peut également que seul le sujet ou seul l'objet soient du pluriel. Le cas d'un sujet pluriel et d'un objet singulier est rare, mais il est néanmoins attesté. Ainsi, quand on lit dans le Midi Libre du 15 février 2008 que «Quatre hommes... peuvent en cacher un autre », ce qu'il faut comprendre c'est que quatre hommes politiques ont tous posé leur candidature pour le poste de maire de Plaissan, s'opposant par là à un cinquième qui reste dans les coulisses et ne s'est pas encore décidé. On notera dans ce titre de presse le recours à un numéral plutôt qu'à l'article indéfini. En général, on ne touche pas à l'article, sauf pour le mettre au féminin ou au pluriel. Il arrive cependant aussi qu'un article défini ou un adjectif démonstratif soient utilisés au lieu d'un article indéfini. Nous en verrons des exemples plus loin et nous contenterons 
ici de rappeler le titre sur le tract du PCF cité au §3.5. : Attention, cette réforme peut en cacher bien d'autres! Ce titre a en outre un sujet singulier et un objet pluriel, cas plus fréquent que celui du sujet pluriel et de l'objet singulier, mais qui reste tout de même rare quand on tient compte de l'ensemble des occurrences de la structure « Un X peut en cacher un autre » :

\author{
Un portraitiste peut en cacher deux autres à Ruffec (La Charente Libre, 17 avril) \\ Une élection peut en cacher d'autres (Midi Libre, 24 avril) \\ Logement social : 1'affaire Bolufer peut en cacher bien d'autres (La Charente Libre, \\ 22 décembre)
}

C'est dans l'usage d'un sujet singulier et d'un objet pluriel, que se manifeste le plus clairement le potentiel d'un abus de la structure. Comment interpréter qu'à Tessin, « demain, un vélo pourra en cacher 2000 autres » (Le Progrès de Lyon, 30 avril 2005 ; à noter également, l'usage du futur simple pourra, auquel nous revenons dans quelques instants) ? C'est une épreuve cycliste qui est annoncée ; tout ce que ce titre de presse cherche à communiquer, c'est qu'on espère atteindre « un record de 2000 participants ».

La productivité de notre structure syntaxique se mesure aussi, nous l'avons dit, au niveau du temps verbal. Le verbe pouvoir peut se mettre à d'autres temps, dont le conditionnel, le futur simple et l'imparfait. L'avertissement de la SNCF ne se prête pas à cette relative souplesse : seul l'indicatif présent y est attesté. La structure existe-t-elle à des temps non envisagés ici ? Si c'est le cas, nous n'en avons pas repéré d'exemples. Quoi qu'il en soit, la thèse de la productivité ne s'en trouverait que renforcée.

Parmi les trois temps autres que l'indicatif présent qui retiendront notre attention, le conditionnel semble être le plus commun. Trois exemples devraient suffire. Hormis le temps verbal, le premier n'a rien d'extraordinaire. La structure syntaxique productive figure dans le titre; un seul paragraphe a été reproduit pour clarifier de quoi il s'agit. Le deuxième exemple, quant à lui, fait intervenir un article défini plutôt qu'indéfini, et le troisième un démonstratif.

\begin{abstract}
Un projet pourrait en cacher un autre...
Gérard Davoine et Virgil estiment qu'avec près de 1800 places, leur multiplexe répond aux attentes locales en matière de cinéma. Ils attendent 600000 entrées par an. C'est cela qui séduit fortement les commerçants, désireux de garder la population (les consommateurs) dans le centre-ville. La capacité du miniplexe d'Eiffage laisse beaucoup plus de chances à un multiplexe périphérique. Le promoteur attend 150000 entrées annuelles avec cet équipement. (Ouest France, 28 mars 2006)

L'affaire pourrait en cacher une autre. Alors que plusieurs personnalités bordelaises sont mises en examen et détenues sous le soupçon d'avoir abusé de la faiblesse de Jeanine Terrasson, riche octogénaire atteinte de la maladie d'Alzheimer, la vieille dame est à son tour montrée du doigt dans une autre procédure : l'enquête, ouverte depuis 2000, vise le détournement de l'héritage de Fernand Laporte, chirurgien girondin mort en 1999 et qui avait fait fortune dans l'immobilier sur le bassin d'Arcachon. (Le Point, 5 avril 2007)

L'eau est une denrée rare. Encore plus concernant la nappe phréatique de Champigny, en rive droite de la Seine. Hier, la préfecture de l'Essonne a indiqué qu'elle appelait à des mesures de restriction de l'usage de l'eau dans 17 communes autour du Val d'Yerres (...). Ces mesures ne touchent pas le réseau « habituel» d'eau potable à la sortie des robinets mais les forages provenant directement de cette nappe phréatique. (...) Mais cette alerte pourrait en cacher une autre... « Si la nappe phréatique et la Seine baissent avec ces très fortes chaleurs, une restriction de l'eau potable pourrait être envisagée, indique Jean-Yves Sommier, le directeur de la Ddaf (Direction départementale de l'agriculture et des forêts). Mais on n'en est pas là. Pour l'instant, c'est un scénario catastrophe que je n'imagine pas. » (Le Parisien, 25 juillet 2008)
\end{abstract}

Miniplexe ou multiplexe (premier exemple) ? Le risque existe que, si un projet de miniplexe plutôt que de multiplexe est adopté pour le centre-ville, un autre projet, à savoir celui d'un multiplexe en périphérie qui éloignera les consommateurs du centre-ville, ne tardera pas à être formulé pour répondre à la demande du public. Par ailleurs, derrière ce qui est désigné dans le deuxième exemple comme une « affaire » se cache 
« une autre procédure », une " enquête » qui est en cours mais qui n'a pas encore mené à une mise en examen ni à une détention. Il y a lieu de croire que si, à la suite de l'enquête, il y a mise en examen et détention, on aura une deuxième affaire. Finalement (troisième exemple), seuls les forages effectués directement dans la nappe phréatique de Champigny font l'objet de l'alerte mise en place par la préfecture. Il n'est cependant pas exclu qu'un jour il y ait aussi des restrictions portant sur l'eau potable « mais on n'en est pas là », c'est-à-dire on ne sait pas encore si cela arrivera ou non.

Passons du conditionnel au futur simple. Pourquoi pourra plutôt que pourrait dans les exemples qui suivent?

Un Crillon pourra en cacher un autre. Après la place de la Concorde depuis 1909, la célèbre enseigne hôtelière va s'afficher au fronton de nouveaux établissements de luxe à Londres, Rome, Barcelone, New York, voire Pékin ou Bombay. (Le Figaro, 24 janvier 2006)

Wally clôturera vendredi soir, la saison du Centre culturel : le bonhomme réinvente le music-hall entre humour et chanson, à la guitare, au piano, ou encore à l'accordéon. (...) Mais, vendredi soir, un spectacle pourra en cacher un autre : le duo Cathon Cataix lui emboitera le pas : un homme, une femme, deux voix, deux accordéons : ces chansonniers toulousains tissent un univers de cabaret gouailleur et poétique, bucolique et réaliste. Et comme tout ce beau monde du Midi se connait depuis belle lurette, Wally les rejoindra sur scène... pour un troisième spectacle ! (Sud Ouest, 22 mai 2007)

Avec le verbe pouvoir au conditionnel, on ne sait pas ce qui arrivera. Seul l'avenir dira si on aura un projet ou deux, une affaire ou deux, une alerte ou deux. Rien de tel ici. Il y aura forcément plusieurs Crillon dès que des établissements du même nom s'ouvriront dans d'autres villes du monde. De la même façon, il y aura plus d'un spectacle au Centre culturel. Cela justifie le recours au futur simple.

Il nous reste à parler des occurrences à l'imparfait, plus nombreuses que celles où s'utilise le futur simple, mais moins nombreuses que les occurrences au conditionnel. ${ }^{18}$ On se contentera de trois exemples :

Pour cette avant-dernière réunion publique de l'année, une bonne nouvelle pouvait en cacher une autre. Et celle d'ouvrir, le 4 décembre, 10 places d'accueil supplémentaire dans un local annexe, à proximité de l'actuelle crèche, en est une pour les parents dont la garde de leur enfant âgé entre 18 mois et 4 ans, est un casse-tête. (Le Progrès de Lyon, 18 novembre 2006)

La semaine dernière, Xavier Breton était arrivé facilement en tête avec 47,24 \% contre $31,98 \%$ à son challenger, mais un tour pouvait en cacher un autre... Finalement, JeanFrançois Debat n'a pas pu refaire son retard. (Le Progrès de Lyon, 18 juin 2007)

Hier, un Sud-Africain pouvait en cacher un autre. Car si on attendait [Percy] Montgomery, on a surtout vu le 3e ligne Gerrie Britz. (L'Indépendant du Midi, 16 décembre 2007)

Trois bonnes nouvelles étaient annoncées lors de la séance publique du conseil municipal dont il est question dans le premier extrait; c'est la troisième qui est introduite à l'aide de l'assertion qu' « une bonne nouvelle pouvait en cacher une autre ». Le deuxième extrait parle des élections législatives dans le département de l'Ain, en 2007. L'assertion Un tour pouvait en cacher un autre se rapporte à un moment où le deuxième tour n'avait pas encore eu lieu; le candidat victorieux avait donc intérêt à ne pas se déclarer élu prématurément. Le troisième extrait décrit un match de rugby à Perpignan. Parmi les joueurs se trouvaient deux Sud-Africains, qui jouaient simultanément, non pas l'un après l'autre. Ce cas est donc différent des deux autres (où les nouvelles et les tours d'élection se sont succédé). On est loin du train qui peut en cacher un autre. 


\subsection{Conséquences de la productivité}

Le plus souvent, quand l'avertissement Un train peut en cacher un autre est adapté aux besoins de celui qui s'en sert, ce sont deux entités indépendantes qui sont en cause, sans aucun rapport entre elles (tout comme, aux passages à niveau, il est question de deux trains entièrement distincts). Il n'en va pas toujours ainsi. La productivité de la structure syntaxique à l'étude a eu plusieurs conséquences. Nous en énumérons quelques-unes.

Tout d'abord, le maintien des articles indéfinis dans la quasi-totalité des occurrences de la structure «Un $\mathrm{X}$ peut en cacher un autre » et l'usage " hors contexte» d'un grand nombre d'entre elles (par exemple dans les titres de presse, quand il faudra lire l'article qui suit pour savoir de quoi exactement il est question) tend à masquer un important glissement sémantique que les occurrences où interviennent un article défini, un adjectif démonstratif ou un numéral d'une part, ou bien un nom propre de l'autre, permettent de mieux apprécier. Quand on se trouve devant un passage à niveau, et qu'on lit sur un panneau qu'un train peut en cacher un autre, il est clair que la lecture qui s'impose est de nature générique. Le sujet un train fait allusion à l'ensemble des trains qui, à un moment ou un autre, pourront passer à l'endroit où le panneau est affiché ; l'objet direct un autre évoque l'ensemble des autres trains qui, immédiatement après, pourront passer au même endroit. Cette lecture générique n'est pas maintenue s'il est question non pas d'une réforme, mais de cette réforme, non pas d'une affaire mais de l'affaire, non pas d'un homme mais de quatre hommes (nous reprenons quelques-uns des exemples cités plus haut). Elle n'est pas maintenue non plus s'il est question d'un Poutine, par exemple, ou d'un Delanoë, etc. En fait, elle n'est même pas maintenue avec l'article indéfini : quand on lit dans le journal qu' « une élection peut en cacher une autre », on ne parle pas d'élections en général, mais d'une élection particulière qui, s'imposant à l'attention de tous, risque de détourner l'attention d'un évènement similaire prévu, par exemple, peu de temps après. Le glissement du générique au spécifique est particulièrement clair si la structure «Un X peut en cacher un autre » s'accompagne d'un complément circonstanciel de temps ou de lieu. Voici un exemple, non encore invoqué dans ce qui précède, où figurent, à côté d'un nom propre, deux compléments circonstanciels et un imparfait :

Hier à la mairie de Biarritz, un Borotra pouvait en cacher un autre. C'est en effet Monsieur frère jumeau, Franck Borotra, qui a remis la médaille de chevalier de la Légion d'honneur à André Trackoen, ancien directeur général des services de la Ville, et actuel président de la SOGICOBA. Pourquoi Franck et pas Didier ? (Sud Ouest, 28 octobre 2005)

La mise en garde implicite dans l'assertion concerne de toute évidence un évènement précis et n'a donc rien de générique. Elle s'adresse rétrospectivement à ceux qui s'étaient imaginé que Didier Borotra ferait les honneurs, alors que c'est son frère jumeau Franck qui a pris sa place. On notera que, même si on enlève les compléments circonstanciels et qu'on mette le verbe au présent, l'assertion reste spécifique : «Un Borotra peut en cacher un autre». On conçoit mal que cet avertissement concerne toutes les personnes qui s'appellent Borotra. Théoriquement, si on remplace le nom propre par un nom commun (p.ex. "Un conseiller peut en cacher un autre»), on pourrait dire que la lecture redevient générique mais ce n'est pas de cette façon-là que la structure productive syntaxique s'utilise en principe : elle s'utilise justement pour parler de situations particulières, et non pas de situations génériques. Il ne sera pas inutile d'ajouter que la perte de la lecture générique en cas de recours à un temps autre que l'indicatif présent résulte dans la perte de la lecture « sporadicité » du verbe pouvoir. Il va sans dire, pour reprendre notre dernier exemple, que si un Borotra pouvait en cacher un autre, hier, à la mairie de Biarritz, il ne le faisait pas «parfois » ou «à certains moments », mais de façon continue et uniquement pour certaines gens, et non pas pour d'autres, jusqu'à ce que tout le monde se rende compte auquel des deux Borotra on avait affaire...

Une deuxième conséquence concerne l'existence indéniable du deuxième $\mathrm{X}$. Le train qui, aux passages à niveau, peut en cacher un autre, très souvent, ne le fait pas. Le cas des emplois dérivés du type «Un X peut en cacher un autre » est différent. En règle générale, on n'utilise la structure syntaxique productive 
que s'il y a effectivement un deuxième $\mathrm{X}$, que le premier cache ou ne cache pas, selon les circonstances. Ceux qui prennent garde ne se feront pas leurrer; par contre, ceux qui sont moins attentifs risquent de ne se douter de rien et de se laisser surprendre. La même chose s'observe quand le verbe pouvoir se met au futur. Nous avons fait remarquer qu'en disant qu'un Crillon pourra en cacher un autre, ou qu'un spectacle pourra en cacher un autre, il y aura effectivement plus d'un Crillon et plus d'un spectacle. Le futur simple ne fait que remettre à plus tard l'existence effective du deuxième $\mathrm{X}$. C'est pareil à l'imparfait : il y avait plusieurs bonnes nouvelles lors de la séance du conseil municipal, il y a eu deux tours dans les élections législatives et il y avait deux Sud-Africains qui jouaient au rugby au même moment. En revanche, l'existence de deux X n'est pas établie si le verbe se met au conditionnel. S'exprimer à ce temps-là revient le plus souvent à dire qu'on ne sait pas s'il y aura un deuxième X ou s'il n'y en aura pas. Le conditionnel pourrait ne préserve qu'une seule des lectures possibles de l'indicatif présent utilisé dans la formule de départ, à savoir la lecture «éventualité » exprimée à l'aide de l'adverbe peut-être. Peut-être qu'il y aura un autre X, peut-être qu'il n'y en aura pas. Un projet, une affaire ou une alerte (nous reprenons une fois de plus les exemples donnés tout à l'heure) qui pourraient en cacher un(e) autre en cachent peut-être un(e) autre. Il n'est pas sûr qu'il y aura une autre alerte (sur l'eau potable), un autre projet (de multiplexe en périphérie) ou une autre affaire (de détournement d'héritage) - tout comme il n'est pas sûr, quand on dit qu'un train peut en cacher un autre, que c'est effectivement le cas, qu'il y en aura un autre.

S'exprimer au conditionnel peut aussi revenir à dire qu'on ne sait pas encore s'il y a (plutôt que s'il y aura) un deuxième $\mathrm{X}$ ou non. C'est ce dernier cas qui est illustré dans l'exemple que voici :

Un Pasqua pourrait en cacher un autre. La semaine dernière, à l'occasion d'une
perquisition en Corse, des policiers ont découvert des documents détaillant des
versements d'argent liquide à plusieurs personnes dont l'identité est masquée par des
pseudonymes. Un versement de 550000 francs ( 83 850 euros) à « Pasqua » apparait
sur un document, comme l'a évoqué le Canard enchaîné. Cette perquisition était
effectuée lors de la garde à vue de Michel Tomi et Robert Feliciaggi, des proches de
Charles Pasqua. Ces deux hommes sont au centre d'une enquête pour «blanchiment
d'argent » menée par un juge monégasque, qui semble croiser celle du juge ajaccien
Jean-Michel Gentil, également pour « blanchiment », concernant le casino d'Ajaccio.
Des soupçons pèsent sur un financement du RPF, le parti de Charles Pasqua, par
Feliciaggi et Tomi. Il est toutefois possible que le «Pasqua » apparu sur ce document
soit un homonyme de l'ancien ministre de l'Intérieur. (Libération, 29 juin 2001)

Le Pasqua dans le document saisi s'appelle peut-être de son prénom Charles (auquel cas il n'y a qu'un seul Pasqua en cause), mais il n'est pas exclu qu'il s'agisse d'un autre Pasqua (auquel cas il y en a deux). C'est dans ce sens qu' " un Pasqua (dont tout le monde a entendu parler) pourrait en cacher un autre, en cache peut-être un autre (que le grand public ne connait pas)». Dans ce cas particulier, et dans celui du projet, de l'affaire et de l'alerte, bref dans tous les cas où on recourt au conditionnel, ce n'est donc pas la lecture « capacité » qui s'impose, ni la lecture «sporadicité », mais la lecture « éventualité ».

Il se peut que le deuxième $\mathrm{X}$, dont nous avons dit qu'en principe il existe indéniablement, se confonde physiquement avec le premier. Ce type de confusion ontologique est bien entendu inimaginable quand on parle d'un train qui peut en cacher un autre. Dans la presse, on l'a vu, on trouve pas mal de titres du genre «Un Delanoë peut en cacher un autre », « Un Alain Landais peut en cacher un autre » etc. Hors contexte, il n'est pas clair s'il s'agit, par exemple, de deux Delanoë différents, de deux aspects différents du même (Bertrand) Delanoë, de deux moments différents dans la carrière politique de ce (Bertrand) Delanoë, etc. Il faut lire l'article qui suit pour le savoir. Aucune de ces possibilités n'est à exclure, ce qui montre qu'en devenant productive la structure syntaxique «Un X peut en cacher un autre» devient également plus flexible : en effet, la séquence originale Un train peut en cacher un autre fait toujours allusion à deux trains différents. Autre exemple: quand on s'occupe de traçabilité alimentaire et que l'on proclame " qu'une viande peut en cacher une autre " (Le journal du CNRS 169, février 2004), il n'est question que d'un seul plat qui, à titre d'exemple, a l'air de foie gras pur, alors qu'il contient des éléments de poulet. 
Nous mentionnerons pour terminer le cas des entités indissolublement liées. Dans « Un problème peut en cacher un autre », texte de P. Bourdieu rédigé en novembre 1989, mais resté inédit jusqu'en 2002, un lien est établi entre un incident isolé, l'exclusion, du collège Gabriel-Hafez à Creil, de trois jeunes filles musulmanes qui insistaient à porter le voile, et une problématique bien plus vaste et bien plus insidieuse :

\begin{abstract}
En projetant sur cet évènement mineur, d'ailleurs aussitôt oublié, le voile des grands principes, liberté, laïcité, libération de la femme, etc., les éternels prétendants au titre de maitre à penser ont livré, comme dans un test projectif, leurs prises de position inavouées sur le problème de l'immigration : du fait que la question patente - faut-il ou non accepter à l'école le port du voile dit islamique ? - occulte la question latente faut-il ou non accepter en France les immigrés d'origine nord-africaine ?-, ils peuvent donner à cette dernière une réponse autrement inavouable. (Bourdieu 2002 : 305)
\end{abstract}

L' " évènement mineur » - qui, soit dit en passant, n'a pas été « aussitôt oublié »- est le corollaire d'une problématique plus vaste; il s'agit de deux entités, certes, mais qui sont indissolublement liées (contrairement aux trains).

\title{
$5 \quad$ Un train peut en cacher un autre : nouveaux contextes d'usage
}

Dans le préambule, il était question de productivité, mais aussi du fait qu'au cours des années un second sens est venu s'ajouter au sens original de la formule Un train peut en cacher un autre. Allusion était faite au déploiement de la formule dans de nouveaux contextes; son support formel n'est donc plus nécessairement celui des panneaux érigés près des passages à niveau. Parmi les nouveaux contextes, il y en a qui concernent de près la SNCF, ou bien où la métaphore du train est développée de diverses façons :

Certains vont même jusqu'à expliquer que cette grève des cheminots qui dure, c'est pain béni pour le pouvoir. Ne détourne-t-elle pas le regard des Français d'un problème bien réel - et très largement partagé celui-ci - de pouvoir d'achat ? Attention, « un train peut en cacher un autre »! (Charente Libre, 20 novembre 2007)

Un train peut en cacher un autre. À peine avait-il lancé un premier convoi de 617 médicaments moins remboursés (35\% et non plus $65 \%$ ) que Jean-François Mattei en met un second sur les rails, avec le même sens de l'effet de surprise. (Ouest France, 25 avril 2003)

Dans d'autres cas, par contre, il n'est question ni de trains ni de quoi que ce soit qui s'y rapporte :

Un train peut en cacher un autre. Et si le tourisme local bénéficie des retombées économiques des festivals Pablo-Casals et Ciné-Rencontres (...), il bénéficie également largement, et depuis maintenant quarante ans, de celles de 1'Universitat catalana d'Estiu, l'Université catalane d'Été. (L'Indépendant du Midi, 30 septembre 2008)

Il y a plus de 25 ans, Domenach (1984:142) parlait déjà de «l'habitude médicale de dire qu'un train peut en cacher un autre ». Cette habitude, qui n'a pas disparu, peut être illustrée à l'aide des extraits que voici :

Un train peut en cacher un autre : ainsi, l'examen d'un abdomen nécessite de palper tous les orifices herniaires; par exemple, ce n'est pas parce qu'on a retrouvé une hernie crurale qu'il n'y a pas de hernie ombilicale associée. (R. Guimbaud \& G. Perlemuter, Gastro-entérologie, Elsevier Masson, ${ }^{4} 2005$, p. 200)

En pédiatrie nous disons souvent « un train peut en cacher un autre». Une maladie grave peut en effet se cacher derrière un symptôme bénin. (P. Le Roux, Homéopitchoun : guide pratique de l'homéopathie pédiatrique, Publibook, 2007, p. 58)

À côté du sens original s'est donc très clairement développé un sens dérivé. L'avertissement de la SNCF est comparable à ces «slogans publicitaires particulièrement réussis [qui] survivent parfois à la réalité leur servant de contexte et la dépassent; ils commencent alors à être cités fréquemment, dans des 
situations très différentes de celle de leur énonciation première » (Schapira $2000: 87$ ) ${ }^{19}$ Un train peut en cacher un autre est désormais aussi une tournure figée, une phrase sentencieuse d'ordre parémique. Duchesne (2003 : 94) fait remarquer que la tournure « a acquis valeur de proverbe, avec le sens de Tout n'est pas bien qui parait bien ou Les apparences sont souvent trompeuses ». Schapira s'exprime de façon analogue : elle parle d'un «nouveau proverbe » dont la naissance est « confirmée par son détournement » (1999 : 99), celui-ci étant « le test et la preuve mêmes de l'obtention du statut proverbial » $(2000: 96){ }^{20}$

Mais peut-on vraiment dire, dans le cas de la tournure Un train peut en cacher un autre, que la phrase sentencieuse se soit développée avant la structure syntaxique productive ? Il n'y a pas que les dictons terme que nous avons utilisé dans notre préambule et qui parait préférable à celui de proverbe pour désigner la phrase sentencieuse Un train peut en cacher un autre - qui se prêtent aux détournements. On pense en particulier, puisqu'il en a été question plus haut, à l'ancienne devise de la revue Paris-Match: Le poids des mots, le choc des photos. Ce n'est pas du tout un dicton (ni une phrase sentencieuse de quelque type que ce soit), et pourtant les détournements ont été très nombreux. Dans le cas qui nous concerne, il est tout à fait concevable que la structure syntaxique productive se soit développée en même temps ou bien avant le dicton, rien qu'à la suite de la familiarité de l'avertissement original. Pour y voir clair, il faudrait entreprendre des dépouillements exhaustifs de corpus extrêmement larges.

Ce qui parait plus facile à établir, par contre, c'est que notre dicton a des propriétés qui ne sont pas sans rappeler celles de la structure syntaxique productive, même s'il semble impossible de faire correspondre au sujet et à l'objet direct deux entités indissolublement liées ou bien ontologiquement confondues (sans doute à cause du lien étroit qui subsiste avec l'avertissement de la SNCF, où il est toujours - et inévitablement - question de deux trains distincts). Par contre, il est intuitivement possible (même si les exemples authentiques sont difficiles à repérer) de mettre le dicton au conditionnel, au futur ou à l'imparfait, manipulation à laquelle les proverbes se prêtent bien moins (Un train pourrait, pourra, pouvait en cacher un autre) ${ }^{21}$ Les écarts formels (p.ex. Certains trains peuvent en cacher d'autres, Un train peut en cacher plusieurs autres) ne semblent pas non plus poser de problèmes.

\section{Explicitations en MSN}

Pour des raisons qui deviendront claires dans quelques instants, la première explicitation ci-dessous portera sur la phrase sentencieuse et les autres sur la structure syntaxique productive.

L'explicitation de la phrase sentencieuse Un train peut en cacher un autre repose sur un gabarit à six volets. $^{22}$ Le volet (a) (STATUT DE SAGESSE POPULAIRE) précise que la phrase sentencieuse explicitée existe depuis longtemps sous cette forme et en annonce le contenu. Le volet (b) (SITUATION RÉCURRENTE) résume la façon de penser de ceux qui ignorent « qu'un train peut en cacher un autre » et rappelle que cette façon de penser reste relativement répandue. Si elle ne l'était pas, l'avertissement de la SNCF ne se serait jamais transformé en phrase sentencieuse : le besoin ne s'en serait pas fait sentir. L'ÉVALUATION (volet c) est immédiatement suivie du CONSEIL que la phrase sentencieuse cherche à véhiculer (volet d). Le volet (e) (ANALOGIE INVOQUÉE) rapproche le conseil véhiculé du sens littéral. Le volet (f) insiste sur le BÉNÉFICE POTENTIEL qui existe pour ceux qui s'efforcent de garder la phrase sentencieuse à l'esprit.

«Un train peut en cacher un autre » (phrase sentencieuse) $=$

(a) depuis longtemps, les gens disent ces mots

STATUT DE SAGESSE POPULAIRE quand ils veulent dire quelque chose comme ça :

(b) «d'autres gens pensent souvent comme ça :

SITUATION RÉCURRENTE je sais tout ce qui arrive

je vois tout

j'entends tout

je sens tout 


\begin{tabular}{|c|c|}
\hline (c) & ce n'est pas bien si les gens pensent comme ça \\
\hline (d) & $\begin{array}{l}\text { c'est mieux si les gens pensent comme ça: } \\
\text { je ne peux pas savoir tout ce qui arrive } \\
\text { je ne peux pas tout voir } \\
\text { je ne peux pas tout entendre } \\
\text { je ne peux pas tout sentir }\end{array}$ \\
\hline (e) & $\begin{array}{l}\text { c'est comme ça : } \\
\text { dans certains endroits, après qu'il y a eu un train }{ }_{[\mathrm{M}]} \text {, il peut y avoir un autre } \operatorname{train}_{[\mathrm{M}]} \\
\text { quand quelqu'un est dans un endroit comme ça, } \\
\text { peut-être que ce quelqu'un ne peut pas voir cet autre train } \\
\text { s'il y a un autre train } \\
\text { si quelqu'un est très près de ce train } \text { àien }_{[\mathrm{M}]} \text {, } \\
\text { il peut arriver quelque chose de très mal à ce quelqu'un » }\end{array}$ \\
\hline (f) & It être bien pour quelqu'un si ce quelqu'un pense parfois à cela BÉNÉFICE POTENTIEL \\
\hline
\end{tabular}

Pour la structure syntaxique productive «Un X peut en cacher un autre », d'autre part, nous proposons deux explicitations distinctes. Elles se ressemblent toutefois de près. Le volet (a) rappelle chaque fois le STATUT DE SAGESSE POPULAIRE du gabarit des phrases sentencieuses ; on y a cependant ajouté le primitif POUVOIR afin d'exprimer l'idée que, justement, ce n'est pas à un morceau de sagesse populaire qu'on a affaire, mais à une simple assertion coulée dans une structure syntaxique productive ; cette assertion porte sur un objet ou une personne quelconques ( « un $X$ », ou bien simplement «X »), ce qu'évoque l'addition en fin de volet de quelques mots. À la fin du volet (a), « au sujet des X » s'impose dans tous les cas où il $\mathrm{y}$ a deux entités distinctes (un $\mathrm{X}$ et un autre $\mathrm{X}$ ). Si X renvoie à un nom propre, et que deux « facettes » de la même personne soient en cause, plutôt que des personnes différentes (confusion ontologique des deux $\mathrm{X})$, il faudra dire « au sujet de $\mathrm{X} »$.

Au volet (b), quasi identique dans les deux cas à la SITUATION RÉCURRENTE dans l'explicitation de la phrase sentencieuse, il est nécessaire d'ajouter une composante supplémentaire « ces gens pensent comme ça quand ils pensent à (des) X ». La présence ou l'absence de l'article indéfini est déterminée par le statut de l'élément qui prend la place du X, comme dans le cas du volet (a).

Le volet (c) est rigoureusement identique à l'ÉVALUATION d'il y a quelques instants. Le volet (d) reprend le CONSEIL VÉHICULÉ, avec une composante supplémentaire ( « il y a deux X » ou bien « je ne sais pas tout au sujet de X »). Il n'y a pas de BÉNÉFICE POTENTIEL; au lieu de l'ANALOGIE INVOQUÉE, on a un volet (e) qui renvoie au rapport, toujours très réel, entre la structure syntaxique productive et l'avertissement original de la SNCF. On aura, selon le cas, « un $X$ » ou bien simplement « $X$ », et toute allusion à « quelque chose de très mal » a disparu.

"Un X peut en cacher un autre » (p. ex. "Une grève peut en cacher une autre »)=

(a) depuis longtemps, les gens peuvent dire ces mots quand ils veulent dire quelque chose comme ça au sujet des $\mathrm{X}$ :

(b) «d'autres gens pensent souvent comme ça :

je sais tout ce qui arrive

je vois tout

j'entends tout

je sens tout

ces gens pensent comme ça quand ils pensent à des $\mathrm{X}$

(c)

ce n'est pas bien si les gens pensent comme ça 
(d) c'est mieux si les gens pensent comme ça :

je ne peux pas savoir tout ce qui arrive

je ne peux pas tout voir

je ne peux pas tout entendre

je ne peux pas tout sentir

il y a deux $\mathrm{X}$ »

(e) quand quelqu'un dit ces mots, ce quelqu'un pense comme ça :

un $\mathrm{X}$ est comme un $\operatorname{train}_{[\mathrm{M}]}$

quand je dis ces mots, je sais que vous pensez la même chose

«Un Xpeut en cacher un autre » (p. ex. "Un (Bertrand) Delanoë peut en cacher un autre ») =

(a) depuis longtemps, les gens peuvent dire ces mots

quand ils veulent dire quelque chose comme ça au sujet de $\mathrm{X}$ :

(b) «d'autres gens pensent souvent comme ça :

je sais tout ce qui arrive

je vois tout

j'entends tout

je sens tout

ces gens pensent comme ça quand ils pensent à $\mathrm{X}$

(c) ce n'est pas bien si les gens pensent comme ça

(d) c'est mieux si les gens pensent comme ça :

je ne peux pas savoir tout ce qui arrive

je ne peux pas tout voir

je ne peux pas tout entendre

je ne peux pas tout sentir

je ne sais pas tout au sujet de $\mathrm{X} »$

(e) quand quelqu'un dit ces mots, ce quelqu'un pense comme ça :

$\mathrm{X}$ est comme un $\operatorname{train}_{[\mathrm{M}]}$

quand je dis ces mots, je sais que vous pensez la même chose

\section{$7 \quad$ Une valeur culturelle à explorer : la méfiance}

Dans ce qui précède, nous avons identifié, décrit et explicité en métalangue sémantique naturelle une structure syntaxique productive du français contemporain, de même qu'un dicton apparenté. Il convient toutefois de rappeler que le but ultime de l'ethnosyntaxe est de voir si, derrière des structures syntaxiques productives propres à un univers culturel particulier, se cachent des valeurs culturelles propres à l'univers en question (Peeters 2009, 2010). Il est relativement simple de vérifier, à partir des nombreux exemples donnés dans ce qui précède, que s'il y a une valeur culturelle sous-jacente à la structure « Un X peut en cacher un autre » et au dicton Un train peut en cacher un autre, ce n'est pas forcément celle de la prudence, valeur qui sous-tend l'avertissement de la SNCF et qui est plus ou moins universelle (quoique sous des guises sans doute légèrement différentes d'un univers culturel à l'autre). En effet, dans la plupart de nos exemples, il n'est pas question de risque physique. Plutôt qu'un appel à la prudence, qualité dont il faut faire preuve en cas de danger physique, la structure syntaxique productive «Un X peut en cacher un autre » et la tournure figée Un train peut en cacher un autre constituent, semble-t-il, un appel à la méfiance (dans laquelle certains seront enclins à voir un défaut plutôt qu'une qualité) : elles impliquent qu'il faut se méfier des apparences, que derrière l'entité (personne ou chose) qui est immédiatement perceptible se cache une entité du même type (ou un aspect de la même entité) dont on ne se rendait peut- 
être pas compte mais qu'il importe de ne pas perdre de vue. Celui qui observe, par exemple, qu'un conflit, ou un maire, ou un Sarkozy, peuvent en cacher un autre, ne nous invite pas à la prudence, car il n'y a pas de danger physique ; il nous dit : «Faites attention, on n'a pas encore tout vu, le pire est à venir ; méfiezvous! » Ce verbe est d'ailleurs explicitement présent dans les extraits que voici :

\begin{abstract}
Mardi matin, les conseillers généraux savaient donc à quoi s'attendre lorsqu'ils ont accueilli le représentant de l'État venu leur faire le bilan de l'action de l'État dans le département. (...) Ils auraient dû se méfier. Un préfet qui commence son intervention en ironisant sur l'opinion si versatile capable de dénoncer le « trop d'État » un jour et d'exiger " plus d'État » le lendemain peut devenir incontrôlable. Même s'il ne visait pas expressément les élus eux-mêmes, le bon mot pouvait en cacher... de moins plaisants. (Ouest France, 28 octobre 2006)

Méfiez-vous : un train de paroles peut en cacher un autre! (L'Humanité, 8 novembre 2006)

D'un côté, vous aurez les toxiques et les mortels. De l'autre, ceux préférés des gourmets. Méfiez-vous, car les uns ressemblent aux autres, un champignon peut en cacher un autre! (Le Progrès de Lyon, 6 octobre 2007)

Le radar de la commune d'Exideuil situé sur la RN 141 dans les virages avant la ligne droite de l'entrée de Chabanais n'en finit pas de s'attirer les foudres des automobilistes. (...) Les automobilistes doivent tout de même se méfier : un radar, même aveugle, peut en cacher un autre... mobile dans l'agglomération chabanoise. (La Charente Libre, 6 janvier 2009)
\end{abstract}

L'association entre les structures du type « Un X peut en cacher un autre » et la méfiance est telle qu'il est question de méfiance jusque dans les cas où l'interprétation est littérale, et en dernière analyse extrêmement proche de l'avertissement original de la SNCF :

Mme Gonon (...) a rappelé aux élèves quelques règles d'or de prudence à respecter, afin de garantir une meilleure sécurité : attendre que le car se soit éloigné avant de traverser ; regarder dans les deux sens avant de s'engager ; traverser sans courir dans les passages piétons ; se méfier, car un véhicule peut en cacher un autre ; rester en arrière à l'arrivée du car; patienter jusqu'à l'arrêt complet du car avant de s'approcher ; ranger son cartable sous le siège ; attacher sa ceinture de sécurité. (Le Progrès de Lyon, 15 octobre 2005)

Quant au rapport entre la structure «Un X peut en cacher un autre » et les apparences (dont il faut se méfier), il se laisse illustrer notamment à l'aide d'un article du Figaro intitulé « Design : un meuble peut en cacher un autre ». Celui-ci s'ouvre de la façon suivante :

Ventes et expositions sont l'occasion de se faire l'œil, d'apprendre, avant de passer à l'action... à l'achat plutôt. Nos conseils pour ne plus vous laisser aveugler par les apparences si vous aimez le design, des pistes pour faire la différence entre des pièces design qui restent similaires aux yeux des néophytes. (Le Figaro, 15 septembre 2006)

Le mot méfiance tombe un peu plus loin :

Une chaise longue, visiblement de fabrication récente, mais dépourvue de cette marque [la signature de Le Corbusier ; B.P.], doit donc susciter la méfiance.

La méfiance constitue-t-elle une valeur culturelle française ? Il y a lieu de croire que oui, mais il faudrait, pour le prouver, entreprendre une étude ethnoaxiologique qui vise à corroborer la réalité d'une valeur culturelle présumée, en invoquant des faits linguistiques aussi bien que non linguistiques qui en constituent le reflet. Ce sera l'objet d'une autre étude, en cours de préparation (Peeters à paraitre).

\title{
Références bibliographiques
}

Domenach, M. (1984). Incertitude et diagnostic médical. In Bayle, J.-J. (éd.), Cultures en conflit ?, Saint-Étienne : Université de Saint-Étienne, 139-152. 
Duchesne, P. (2003). La lumière des mots : quand les proverbes nous éclairent. Namur : Les éditions namuroises.

Fuchs, C. (éd.). (1989). Modalité et interprétation : pouvoir. Langue française, 84.

Fuchs, C. (1991). Polysémie, interprétation et typicalité : l'exemple de «pouvoir ». In Dubois, D. (éd.), Sémantique et cognition : catégories, prototypes, typicalité. Paris : Éditions du CNRS, 161-170.

Goddard, C. (éd.). (2008). Cross-linguistic semantics. Amsterdam : John Benjamins.

Goddard, C. (2010a). The Natural Semantic Metalanguage approach. In Heine, B., Narrog, H. (éd.), The Oxford handbook of linguistic analysis. Oxford : Oxford University Press, 459-484.

Goddard, C. (2010b). «Like a crab teaching its young to walk straight » : proverbiality, semantics and indexicality in English and Malay. In Senft, G., Basso, E. (éd.), Ritual communication. Oxford : Berg.

González Vázquez, M. (2000). Les traits de potentialité et de condition dans l'indétermination de pouvoir. In Englebert, A., Pierrard, M., Rosier, L. \& van Raemdonck, D. (éd.), Actes du XXIIe Congrès international de linguistique et de philologie romanes. Vol. 7. Tübingen : Niemeyer, 277-285.

Grésillon A. ; Maingueneau, D. (1984). Polyphonie, proverbe et détournement, ou : un proverbe peut en cacher un autre. Langages, $73,112-125$.

Honeste, M.-L. (2004). Langue et contexte : deux sources de signification. L'exemple du verbe modal pouvoir. Le français moderne, $72,146-156$.

Jonasson, K. (1994). Le nom propre : constructions et interprétations. Louvain-la-Neuve : Duculot.

Kleiber, G. (1983). L’emploi « sporadique» du verbe pouvoir en français. In David, J. \& Kleiber, G. (éd.), La notion sémantico-logique de modalité. Paris : Klincksieck, 183-203.

Le Querler, N. (2001). La place du verbe modal pouvoir dans une typologie des modalités. Cahiers Chronos, 8, 1732.

Peeters, B. (éd.). (2006). Semantic primes and universal grammar : empirical evidence from the Romance languages. Amsterdam : John Benjamins.

Peeters, B. (2009). Language and cultural values: the ethnolinguistic pathways model. Fulgor, 4, 59-73 (http://ehlt.flinders.edu.au/deptlang/fulgor/).

Peeters, B. (2010). La métalangue sémantique naturelle: acquis et défís. In François, J. (éd.), Grandes voies et chemins de traverse de la sémantique cognitive. Leuven : Peeters, 75-101.

Peeters, B. (à paraitre). Langue française, valeurs françaises : pour une approche des valeurs culturelles à travers la langue.

Schapira, C. (1999). Les stéréotypes en français : proverbes et autres formules. Paris : Ophrys.

Schapira, C. (2000). Proverbe, proverbialisation et déproverbialisation. Langages, 139, 81-97.

Sueur, J.-P. (1977a). Quantificateurs et modalités. Langages, 48, 84-99.

Sueur, J.-P. (1977b). À propos des restrictions de sélection: les infinitifs devoir et pouvoir. Lingvisticae investigationes, 1, 375-409.

Sueur, J.-P. (1979). Une analyse sémantique des verbes devoir et pouvoir. Le français moderne, 47, 97-120.

Sueur, J.-P. (1983). Les verbes modaux sont-ils ambigus? In David, J. \& Kleiber, G. (éd.), La notion sémanticologique de modalité. Paris : Klincksieck, 165-182.

Vetters, C. (2007). L’emploi « sporadique » de pouvoir est-il aléthique ?. Cahiers Chronos, 19, 63-78.

Wierzbicka, A. (1972). Semantic primitives, Frankfurt : Athenäum.

Wierzbicka, A. (1979). Ethno-syntax and the philosophy of grammar. Studies in language, 3, 313-383.

Wierzbicka, A. (2006). Sens et grammaire universelle: théorie et constats empiriques. Cahiers Ferdinand de Saussure, 59, 151-172. 


\begin{abstract}
Notes
${ }^{1}$ Nous incluons dans notre étude l'ensemble des structures du type « Dét. X \{pouvoir\} en cacher Dét. autre(s) », mais recourons à la paraphrase «Un X peut en cacher un autre » pour des raisons de lisibilité. Dét. inclut les numéraux et autres expressions de quantité. De nombreuses autres constructions formellement apparentées n'ont pas été examinées. On pense notamment aux constructions où le verbe pouvoir fait défaut («Un X en cache un autre ») ou est remplacé par un autre verbe (sembler, ne pas devoir). Il y a aussi des constructions où le verbe cacher est remplacé par un synonyme (voiler, dissimuler). Elles n'ont pas été envisagées dans la mesure où le rapport avec l'avertissement de la SNCF est moins net, et peut-être tout simplement inexistant.
\end{abstract}

${ }^{2}$ Aux yeux de Jonasson (1994 : 214), le nom propre dans l'énoncé Un Michel peut en cacher un autre (il est question de Michel Drucker et de Michel Denisot, deux animateurs de télévision) « sert de base de comparaison, donnant naissance à des analogies et à des effets de contraste, à des transitions et à des oppositions, tout cela contribuant à la cohérence d'un texte ordonné ». Elle n'envisage donc que l'une des trois possibilités notées ici.

${ }^{3}$ Sur le verbe pouvoir en français, et en nous limitant aux études publiées depuis les années quatre-vingts, signalons Kleiber (1983), Fuchs (1989, 1991), González Vázquez (2000), Le Querler (2001), Honeste (2004) et Vetters (2007). La plupart de ces études renvoient aux premiers travaux de Jean-Pierre Sueur (p.ex. Sueur 1977a, 1977b, 1979, 1983), références devenues aujourd'hui quasi incontournables.

${ }^{4}$ « Possibilité matérielle » renvoie à des circonstances externes telles que la visibilité ou le temps qu'il fait.

${ }^{5}$ Plutôt que THE SAME (voir la justification dans Peeters 2006).

${ }^{6}$ Plutôt que TU, proposé dans Peeters (2006). Pour une justification, voir Peeters (2009a). Anna Wierzbicka (communication privée, janvier 2009) nous a signalé que, s'il s'avère que $t u$ ne se laisse pas définir de façon adéquate en termes du primitif vous, ni vous en termes du primitif TU, il faudra traiter TU et vous comme des allolexes. Quoi qu'il en soit, il ne s'agit certainement pas de deux primitifs distincts.

${ }^{7}$ Peeters (2006) proposait IL Y A ... QUI, comparable à la version italienne C’è ... CHE. Si nous revenons aujourd'hui à une lexicalisation proposée dans des écrits antérieurs, c'est que IL Y A ... QUI ne se prête pas à la même combinatoire.

${ }^{8}$ Plutôt que POUR UN TEMPS, proposé dans Peeters (2006), et qui parait moins naturel.

${ }^{9}$ Les phrases canoniques illustrent également le fait que certains primitifs se lexicalisent de plusieurs façons distinctes, selon le contexte. Les variantes en question s'appellent des allolexes. Ainsi, ME et MOI sont des allolexes de JE. ENDROIT, pour donner un autre exemple, est un allolexe d'ou, utilisé quand ce primitif se combine avec un déterminant ou un quantificateur (CET ENDROIT, UN AUTRE ENDROIT, BEAUCOUP D'ENDROITS). Le primitif QUELQUE CHOSE a plusieurs allolexes : CHOSE après des déterminants et des quantificateurs (on aura donc TOUTES CES CHOSES, et non pas *TOUS CES QUELQUES CHOSES), RIEN dans des contextes négatifs, CE QUI et CE QUE dans des questions indirectes et des propositions relatives. Le primitif QUAND, quant à lui, en a trois (QUAND, MOMENT et FOIS). On pourrait multiplier les exemples.

${ }^{10}$ Sur la notion de molécule sémantique, on verra notamment Goddard (2010a) et, en français, Peeters (2010). À l'instar des autres molécules, $\operatorname{train}_{[M]}$ devra en principe faire l'objet d'une description rigoureuse d'où rien d'essentiel ne soit omis. C'est une tâche à laquelle, faute d'espace, nous ne nous attèlerons pas. Disons simplement qu'une description en primitifs sémantiques, sans recours à d'autres molécules, risque d'être impossible. Il faudra sans doute prévoir une deuxième molécule, rail $_{[M]}$, dont l'explicitation nécessitera d'autres molécules encore, notamment long $[M]$. 
${ }^{11}$ Un plus joli témoignage de l'importance que revêt l'avertissement de la SNCF dans l'univers culturel français serait difficile à trouver.

${ }^{12}$ Orizet n'est pas le seul - il s'en faut de loin - à faire précéder les structures du type « Un X peut en cacher un autre » du mot attention. Les panneaux de la SNCF ne l'incluent pas.

${ }^{13}$ Il arrive aussi que la formule Un train peut en cacher un autre soit associée à une formule du type « Un $\mathrm{X}$ peut cacher un Y ». Deux exemples suffiront : " Comme un train peut en cacher un autre, un titre peut cacher une pièce » (Le Monde, 1 septembre 2005) ; "Comme un train peut en cacher un autre, une grève peut cacher une révolution syndicale » (Midi Libre, 23 novembre 2007).

${ }^{14}$ L'avenir dira si la nouvelle devise de l'hebdomadaire Paris Match, " La vie est une histoire vraie », pourra faire oublier l'ancienne. Celle-ci a fait fortune depuis sa création en 1976, mais on l'a si souvent détournée que la rédaction a vraisemblablement voulu tourner la page. Kermoal (art.cit.) reproduit trois exemples (auxquels on peut ajouter, parmi plusieurs autres, celui qu'il propose lui-même dans son titre), à savoir Le poids des pots, le choc des motos (presse auto), Le poids des beaux, le choc des héros (presse féminine), et Le poids des mots, le choc des campagnes (magazine de communication).

${ }^{15}$ Sauf indication contraire, tous les exemples cités dans ce qui suit sont des titres d'articles de presse (journaux et hebdomadaires) publiés en 2007. Il y en a des dizaines.

${ }^{16}$ La présence du pronom relatif qui témoigne de la flexibilité de la structure, flexibilité qui, elle, est le résultat direct de sa productivité. Il y a trop peu d'exemples pour y revenir ci-dessous.

${ }^{17}$ Dans le cas de mots polysyllabiques (chrétien [Midi Libre, 31 janvier], pèlerin [Centre Presse, 18 octobre], jardin [Le Progrès de Lyon, 2 novembre], Sud-Africain [L'Indépendant du Midi, 16 décembre]), l'effet de la rime est nettement moindre.

${ }^{18}$ Les cas où c'est la concordance des temps qui impose l'imparfait (cas du type « Il était clair qu'un X pouvait en cacher un autre ») n'ont pas été envisagés.

${ }^{19}$ On comprend moins bien que Schapira ait choisi d'exemplifier cette thèse relative au sort de certains slogans publicitaires à l'aide de la tournure Un train peut en cacher un autre, comme s'il s'agissait d'un slogan plutôt que d'un avertissement... Dans un autre texte, elle avait compté la tournure parmi les «slogans dont l'origine s'est estompée » (Schapira 1999: 129), ce qui ne semble pas non plus correspondre à la réalité (voir $\S 2$ ci-dessus).

${ }^{20}$ L'auteure signale à ce sujet, à côté d'exemples comparables aux dizaines qui figurent dans ces pages, le sous-titre («Un proverbe peut en cacher un autre ») d'un article savant (Grésillon \& Maingueneau 1984 ; cf. Schapira $1999: 99,153 ; 2000: 96)$.

${ }^{21} \mathrm{Cf}$. *Qui ne dirait mot, consentirait ; *Qui ne dira mot consentira ; *Qui ne disait mot consentait. Au discours indirect, on imagine facilement: J'ai toujours su qu'un train pouvait en cacher un autre, mais non ?J'ai toujours su que qui ne disait mot consentait (on garderait plutôt le présent).

${ }^{22}$ Le gabarit sémantique utilisé ci-après pour l'explicitation des phrases sentencieuses s'inspire de celui proposé par Goddard (2010b), mais s'en écarte en même temps sur plusieurs points. 